\title{
STEEL DOME PROJECT DESIGN BOGOR CONVENTION CENTER OPTIMIZATION FOR BUDGET AND AESTHETIC ARCHITECTURE
}

\author{
Hamonangan, GIRSANG \\ Faculty of Engineering, \\ University of Mercu Buana Jakarta, Indonesia \\ hamongirsang@gmail.com \\ Cecep Indra, LESMANA \\ Faculty of Engineering, \\ University of Mercu Buana Jakarta, Indonesia \\ chep_indra@ymail.com
}

\begin{abstract}
A building needs to be considered various aspects to meet the values - both economic value, cost, durability, impact on the environment to aesthetics or beauty. This research was conducted to make a comparison of the Bogor Convention Center project which was built using the Dome structure with the WF Steel Profile which will be replaced by using steel pipe material. The software used in designing steel is SAP2000 Analysis. SAP2000 is a 3D modeling application that is able to design steel structures that produce analysis and calculation results include static analysis, finite element dynamics analysis, as well as images, reports or other outputs from one structural model. As for the depiction using Autocad where Autocad functions to detail the connections that are in the dome frame of the study. Analysis and Design of a spherical dome structure with building functions as a convention center. The structure of the shell is used as the roof of the building so that the aesthetic elements and values of the architecture of the shell structure can be seen its beauty from the inside of the building. The analysis conducted is an analysis of the internal forces and design of reinforcement in the structure..
\end{abstract}

Keywords: Dome, Aesthetics, WF Steel, Steel Pipes, Architecture

\section{INTRODUCTION}

Structure dome / shell has architectural value and high art. The inside of the shell structure shows the shape of a beautiful arch and spans a column in it is designed with a large span so as to provide a space that is open and large at the bottom of the shell structure. This provides more value for the use of the room below. Shell structure is used as the roof of the building in order to aesthetics and architectural value of the shell structure can be seen the beauty of the inside of the building. Analysis is conducted analysis of internal forces and reinforcement design in the structure. At first the center of Bogor conventional project using dome structure with Profiles Steel WF and the present study will try to replace the material of the steel profile WF into Steel Pipe. As well as to compare the value of the aesthetic and economical prices for conventional building center Bogor.

Figure 1. The steel dome roof framework convention center

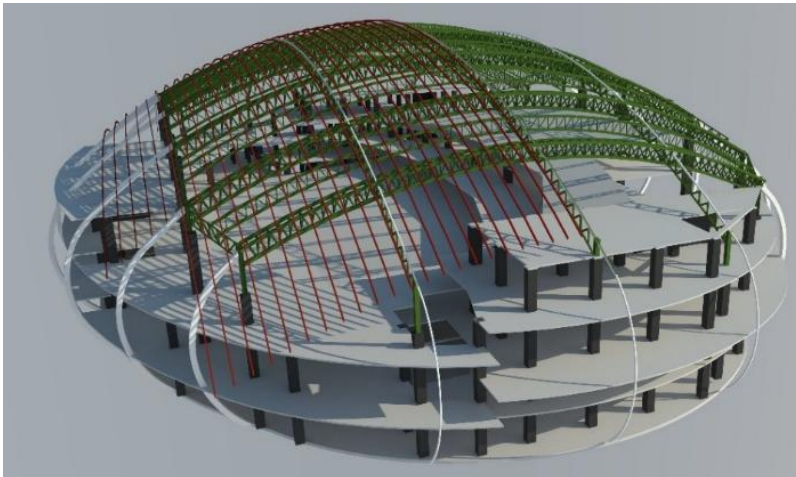

Source: Data from the convention center project 
Geodesic dome structure was first built by Zeiss Optical Company with head engineer Walter Bauersfeld in 1926. But who popularized the geodesic dome structure is Richard Buckminster Fuller, an architect, designer, writer, and scientist. Fuller is the man who coined the term "geodesic" in the dome structure in 1948.

The expenses incurred on the geodesic dome is the axial load tap and drag. Both types of loads that occur in every frame triangles that form the structure of the geodesic dome [2], Expenses incurred in the framework of the triangle can be seen in the following figure:

Figure 2.Triangular load distribution frame geodesic dome

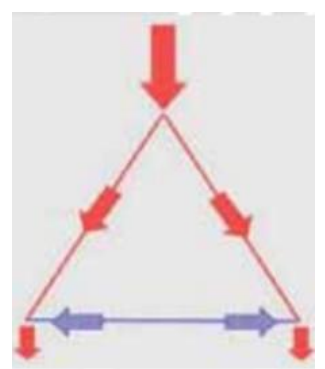

Source:Fahrurrazzi 2011
Figure 3.The load distribution of the

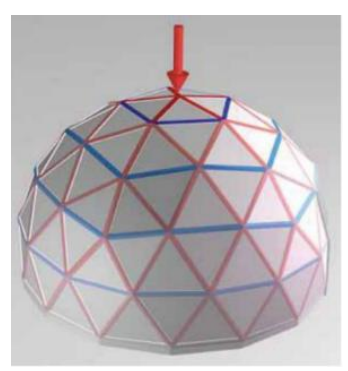

Source:Fahrurrazzi 2011

\section{RESEARCH METHODOLOGY}

The method used in this research is descriptive method with case study (case study). Is a case study research on the status of research subjects that relate to a specific phase or typical of the whole personality. The purpose is to give an overview of case studies in detail about the background, nature and typical of a case characterized the later of the above characteristics will be a matter of a general nature. In accordance with the background research and the formulation of the problem to be achieved then the required primary data and secondary data. From the data collected will be processed and produce data analysis. From the results of this analysis can be concluded as well as suggestions that will answer the purpose of this study. 
Figure 3.Flowchart of Research

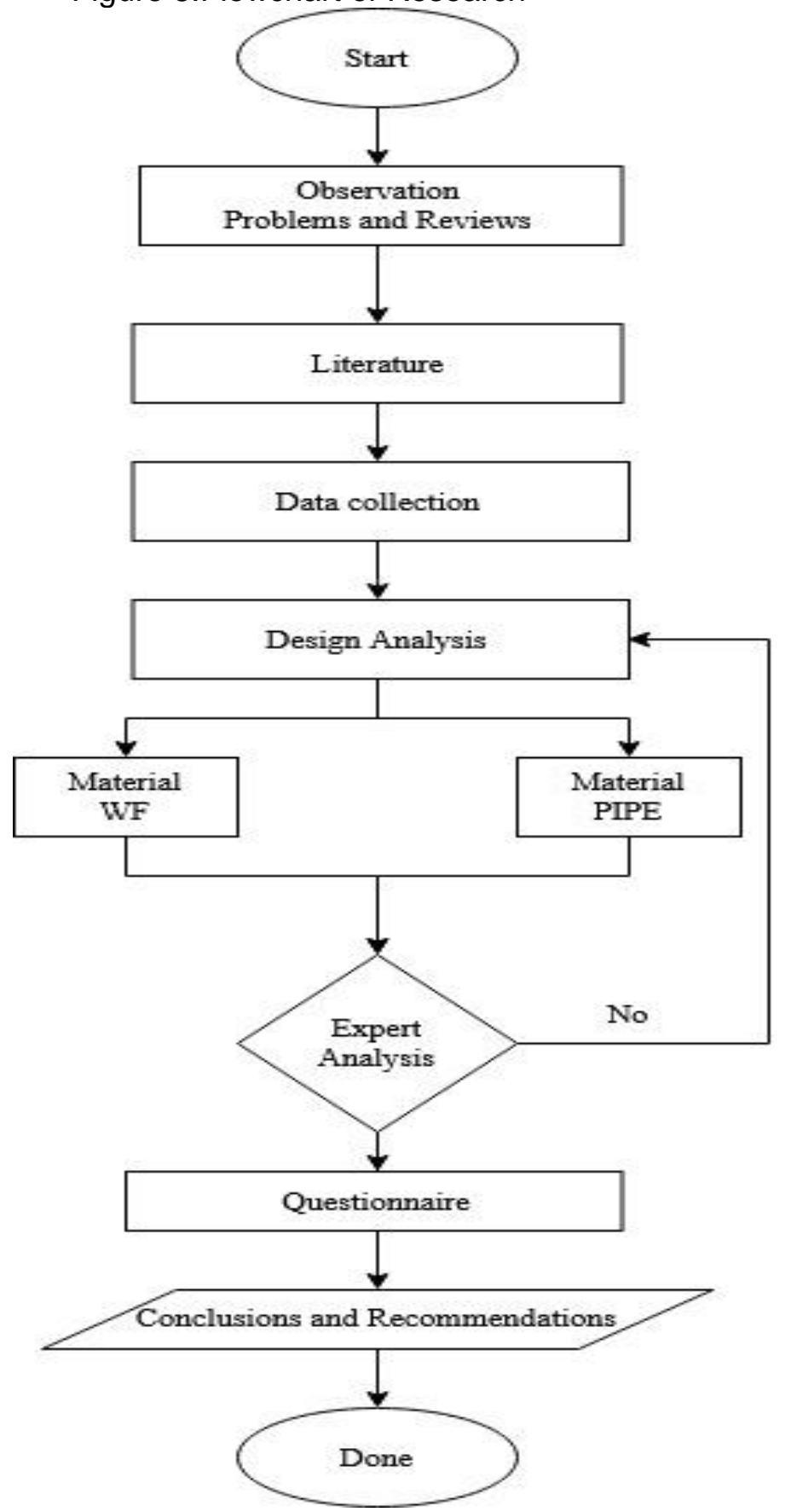

Source: researchers output ,2019

\section{RESULTS AND DISCUSSIONS}

\section{Early Design}

a. Preliminary design Frame Dome

Steel framework of this thesis have the symmetry, where each element in the same order saddled with the same style. From Figure 4 it can be seen that:

$\mathrm{A}=\mathrm{H}, \mathrm{B}=\mathrm{G}, \mathrm{C}=\mathrm{F}, \mathrm{D}=\mathrm{E}$

Thus, in the calculation only reviewed one frame only[3], 
Figure 4.Layout Framework Definition Design Dome Early

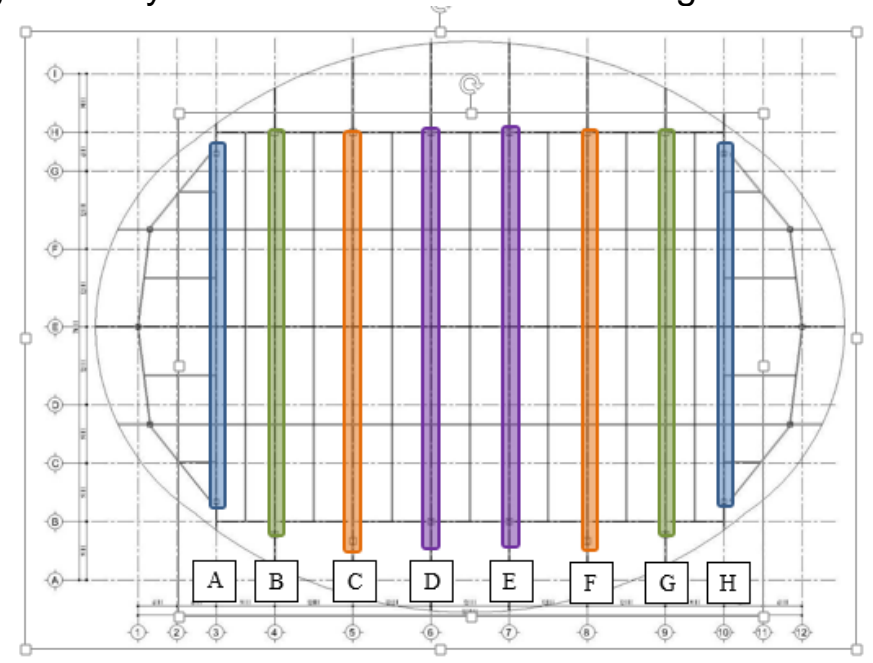

Source: project documentation ,2019

b. Imposition Trunk under review

1. Dead load (DL)

Dead load Evenly $=15 \mathrm{~kg} / \mathrm{m}$

2. Living Expenses (LL)

Centralized Living Expenses - Expenses Person (P-II)

$=100,00 \mathrm{~kg}$

3. Expenses Rain

$R=0.0098(d s+d n)$

$=5.2 \times(0,020+0,025)$

$=0.230 \mathrm{Kg} / \mathrm{m}$

So that the load per meter is

q - Rain

$=\mathrm{RX}$ Length Loading

$=0.23 \times 63.27$

Number $q$ - $R$

$=14.55 \mathrm{Kg} / \mathrm{m}$

4. Wind load

Another structure design wind force should not be less than $0.77 \mathrm{kN} / \mathrm{M} 2$ multiplied by Af

$q=$ Wind $=0.77 \times$ af

q - Wind Total

$=0.77 \times 1 \times 1=0.77 \mathrm{kN} / \mathrm{m}^{2}$

Q Number - Wind Total

$=$ Wind Koef $X \mathrm{q}-$ Wind

$=3 \times 0.77$

$=2.31 \mathrm{kN} / \mathrm{m}^{2}$

c. Early Dome Frame Structure Analysis

Figure 5.Results of Preliminary Design Calculation SAP

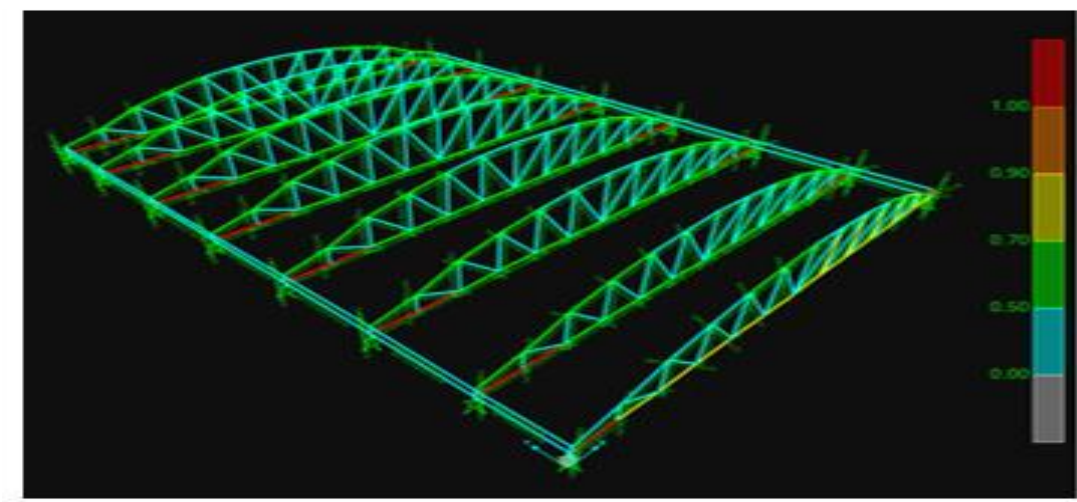

Source: project documentation ,2019 
SAP peritungan of the results can be seen there are a few stems PM ratio is more than 1 (one), it can lead to failure of the profile, so that the initial structure design can not be used on the Project Convention Center in Bogor and needed a new design with the PM ratio below 1. Then made a final design.

\section{Final Design}

a. Frame Design Dome End

Steel framework of this thesis have the symmetry, where each element in the same order saddled with the same style. From figure 4.9 it can be seen that:

$\mathrm{A}=\mathrm{H}, \mathrm{B}=\mathrm{G}, \mathrm{C}=\mathrm{F}, \mathrm{D}=\mathrm{E}$

Thus, in the calculation only reviewed one frame only

Figure 4.Layout Framework Definition Design Dome End

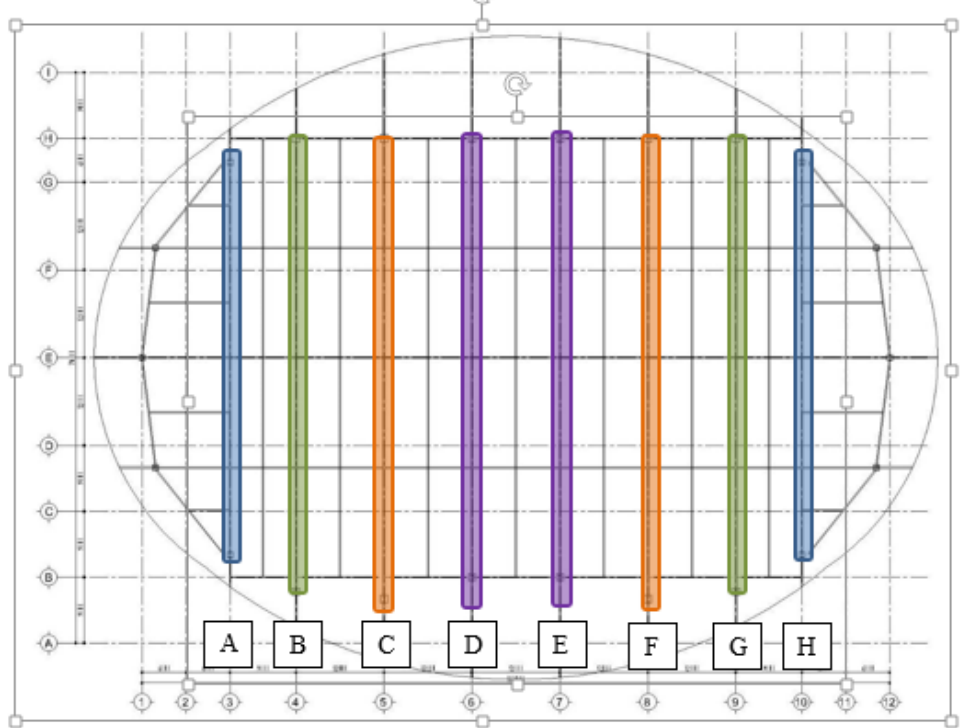

Source: researchers output ,2019

b. Imposition Trunk under review

1. Dead load (DL)

Dead load Evenly $=15 \mathrm{~kg} / \mathrm{m}$

2. Living Expenses (LL)

Centralized Living Expenses - Expenses Person (P-II)

$=100,00 \mathrm{~kg}$

3. Expenses Rain

$R=0.0098(d s+d n)$

$=5.2 \times(0,020+0,025)$

So that the load per meter is

q - Rain

$=0.230 \mathrm{Kg} / \mathrm{m}$

Number $q$ - $R$

$=\mathrm{RX}$ Length Loading

$=0.23 \times 63.27$

$=14.55 \mathrm{Kg} / \mathrm{m}$

4. Wind load

Another structure design wind force should not be less than $0.77 \mathrm{kN} / \mathrm{M} 2$ multiplied by Af

$q=$ Wind

$$
=0.77 \times \text { af }
$$

q - Wind Total

$=0.77 \times 1 \times 1=0.77 \mathrm{kN} / \mathrm{m}^{2}$

$=$ Wind Koef $X \mathrm{q}-$ Wind

Q Number - Wind Total

$=3 \times 0.77$

$=2.31 \mathrm{kN} / \mathrm{m}^{2}$

c. Dome Final Frame Structure Analysis

Figure 6.Calculation of Final Results of SAP Design 


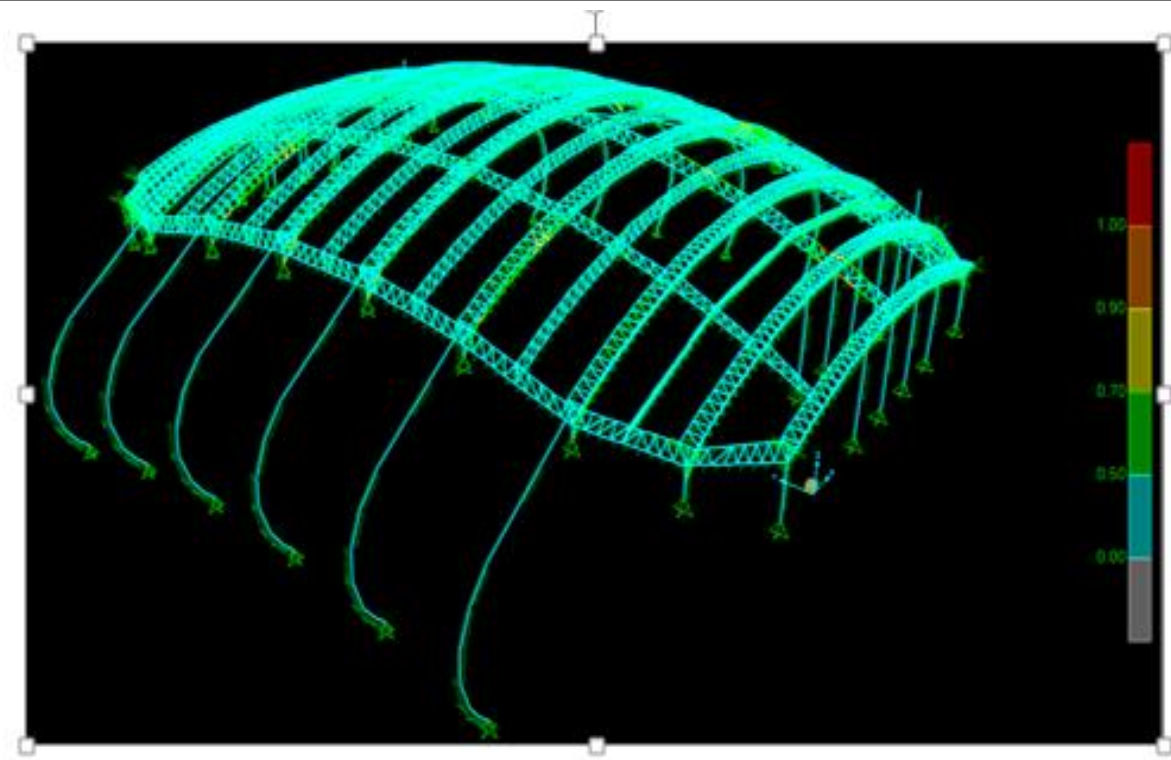

Source: researchers output ,2019

SAP peritungan of the results can be seen there are a few stems PM ratio below 1 (one). Thus this design can be used.

\section{Estimated Cost Analysis}

a. Analysis Preliminary Design Cost Estimation

Figure 6.Preliminary Cost Estimates Table Design

\begin{tabular}{|c|c|c|c|c|c|c|}
\hline \multirow{2}{*}{ No } & \multirow{2}{*}{ URAIAN } & \multirow{2}{*}{ SAT } & \multicolumn{3}{|c|}{ DESIGN AWAL } & \multirow[t]{2}{*}{ KET } \\
\hline & & & VOL & HRG SAT & JML & \\
\hline & & & & & & \\
\hline \multirow[t]{22}{*}{1} & PEKERJAAN STRUKTUR BAJA ATAP & & & & & \\
\hline & & & & & & \\
\hline & Pekerjaan Instalasi \& Pabrkasi Baja Atap, menggunakan Baja BJ & & & & & \\
\hline & fy 250 Mpa, dilaksanakan sesuai spesfikasi dan gambar & & & & & \\
\hline & HB $400 \times 400 \times 13 \times 12$ & $\mathrm{Kg}$ & $10.576,28$ & 42.160 & 445.895 .965 & \\
\hline & HB $300 \times 300 \times 10 \times 16$ & $\mathrm{Kg}$ & $143.448,05$ & 42.160 & 6.047 .769 .785 & \\
\hline & 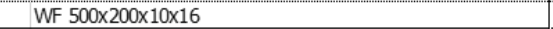 & $\mathrm{Kg}$ & $196.321,78$ & 44.210 & 8.679 .385 .737 & \\
\hline & WF $400 \times 200 \times 8 \times 3$ & $\mathrm{Kg}$ & $182.890,56$ & 44.210 & 8.085 .591 .602 & \\
\hline & GNP $100 \times 50 \times 20 \times 3,2$ & $\mathrm{Kg}$ & $28.074,29$ & 40.310 & 1.131 .674 .549 & \\
\hline & PLATE, T $=25$ & $\mathrm{Kg}$ & $10.370,01$ & 40.000 & 414.800 .531 & \\
\hline & PLATE, $T=15$ & $\mathrm{Kg}$ & $23.485,94$ & 40.000 & 939.437 .760 & \\
\hline & PLATE, $T=10$ & $\mathrm{Kg}$ & $7.952,44$ & 40.000 & 318.097 .524 & \\
\hline & PLATE, $\mathrm{T}=8$ & $\mathrm{Kg}$ & 354,49 & 40.000 & 14.179 .738 & \\
\hline & Anchor $M-20$ & PCS & 440,00 & 210.000 & 92.400 .000 & \\
\hline & Bot M20 (3/4" L 3") I & PCS & $4.070,00$ & 26.000 & 105.820 .000 & \\
\hline & GROUTING, $\mathrm{T}=35$ & M3 & 1,85 & 11.744 .000 & 21.719 .696 & \\
\hline & Cat finishing 2 x 35 micron ex Danapaint & $\mathrm{Kg}$ & $603.473,84$ & 4.200 & 2.534 .590 .136 & \\
\hline & & & & & & \\
\hline & & & & & & \\
\hline & 2.4 - PEKERJAAN STRUKTUR BAJA & & & & 28.831 .363 .023 & \\
\hline & Dipndahkan ke Rekapitulasi Akhir Bill No. 2.4 & & & & & \\
\hline & & & & & & \\
\hline
\end{tabular}

Source: project documentation ,2019

Estimated Cost Design Dome old Steel Works is RP. 28,831,363,023 (TwentyEight Billion Eight Hundred Thirty-One Million Three Hundred Sixty Three Thousand and Twenty-Three Rupiah).

b. Final Design Cost Estimation Analysis 
Figure 7. Final Design Cost Estimation Table

\begin{tabular}{|c|c|c|c|c|c|c|}
\hline \multirow{2}{*}{ No } & \multirow{2}{*}{ URAIAN } & \multirow{2}{*}{ SAT } & \multicolumn{3}{|c|}{ DESIGN AKHIR } & \multirow[t]{2}{*}{ KET } \\
\hline & & & VOL & HRG SAT & JML & \\
\hline & & & & & & \\
\hline \multirow[t]{24}{*}{1} & PEKERJAAN STRUKTUR BAJA ATAP & & & & & \\
\hline & & & & & & \\
\hline & Pekerjaan Instalasi \& Pabrkasi Baja Atap, menggunakan Baja BJ & 41 & & & & \\
\hline & fy $250 \mathrm{Mpa}$, dilaksanakan sesuai spesfikasi dan gambar & & & & & \\
\hline & Profil Baja WF 500.200 & $\mathrm{Kg}$ & $50.752,72$ & 44.210 & 2.243 .777 .788 & \\
\hline & Profil HB 400.400 & $\mathrm{Kg}$ & $10.576,28$ & 42.160 & 445.895 .965 & \\
\hline & Gording Pipa Baja SCH 40 - Dia 3"' & $\mathrm{Kg}$ & $57.679,90$ & 38.830 & 2.239 .710 .548 & \\
\hline & $\mathrm{SCH} 40-\mathrm{P} 4^{\prime \prime}$ & $\mathrm{Kg}$ & $135.294,95$ & 39.190 & 5.302 .209 .106 & \\
\hline & $\mathrm{SCH} 40-\mathrm{P}_{6}^{\prime \prime}$ & $\mathrm{Kg}$ & $27.664,54$ & 39.320 & 1.087 .769 .870 & \\
\hline & $\mathrm{SCH} 40-\mathrm{P}^{\prime \prime}$ & $\mathrm{Kg}$ & $52.225,89$ & 39.400 & 2.057 .700 .145 & \\
\hline & $\mathrm{SCH} 40$ - $\mathrm{P} 10^{\prime \prime}$ & $\mathrm{Kg}$ & $59.545,73$ & 40.280 & 2.398 .501 .924 & \\
\hline & $\mathrm{SCH} 40-\mathrm{P} 12^{\prime \prime}$ & $\mathrm{Kg}$ & $39.378,64$ & 40.430 & 1.592 .078 .253 & \\
\hline & Stiffener Gording Plat Baja T 8 & $\mathrm{Kg}$ & 354,49 & 40.000 & 14.179 .738 & \\
\hline & Plat Baja T 10 & $\mathrm{Kg}$ & $6.468,79$ & 40.000 & 258.751 .524 & \\
\hline & Plat Baja T 15 & $\mathrm{Kg}$ & $5.682,14$ & 40.000 & 227.285 .760 & \\
\hline & Plat Baja T 25 & $\mathrm{Kg}$ & $10.370,01$ & 40.000 & 414.800 .531 & \\
\hline & Angkur Baja M20 L $=60 \mathrm{~cm}$ & PCS & 440,00 & 210.000 & 92.400 .000 & \\
\hline & Bot M20 $\left(3 / 4^{\prime \prime} L 3^{\prime \prime}\right) \backslash$ & PCS & $1.550,00$ & 26.000 & 40.300 .000 & \\
\hline & Grouting $\mathrm{T}=35 \mathrm{~mm}$ & M3 & 1,85 & 11.744 .000 & 21.719 .696 & \\
\hline & Cat finishing $2 \times 35$ micron ex Danapaint (Provisional) & $\mathrm{Kg}$ & $455.994,09$ & 4.200 & 1.915.175.182 & \\
\hline & & & & & & \\
\hline & & & & & & \\
\hline & 2.4 - PEKERJAAN STRUKTUR BAJA & & & & 20.352 .256 .029 & \\
\hline & Dipndahkan ke Rekapitulasi Akhir Bill No. 2.4 & & & & & \\
\hline
\end{tabular}

Source: researchers output, 2019

Estimated Cost Design Dome old Steel Works is RP. 20,352,256,029 (Twenty Billion Three Hundred Fifty Two Million Two Hundred and Fifty Six Thousand Twentynine Rupiah).

\section{CONCLUSION}

From the results of Project Design, Steel Dome Convention Center in Bogor can be concluded as follows:

1. Design Dome initial use Profiles Steel WF as a structural material, with appropriate modeling tender image. After analysis of structure, obtained PM ratio exceeds 1 (one), this can cause failure which led to design profile can not be used.

2. Estimated price Structural Work on the preliminary design produces PricesRP. 28,831,363,023 (Twenty-Eight Billion Eight Hundred Thirty-One Million Three Hundred Sixty Three Thousand and Twenty-Three Rupiah).

3. After looking from the side Structural Strength, Estimated Price \& Aesthetics, then dicobalah separately redesign the frame using the Profile Pipe Steel as a structural material. After dikalukan anailsa structure, didaptlah PM ratio below 1 (one), thus the final design can be used.

4. Estimated price Structural Work on the final design resulted in price RP. 20,352,256,029 (Twenty Billion Three Hundred Fifty Two Million Two Hundred and Fifty Six Thousand Twenty-nine Rupiah). This value is smaller than the initial design to the value of savings ofRP. 8,479,106,994 (Eight Billion Four Hundred Seventy Nine Million One Hundred Six Thousand Nine Hundred Ninety Four Rupiah).

5. Design end rated better meet the aesthetic value to the shape of the structure is not rigid.

\section{REFERENCES}

[1] Kristanti, V., Putranto, AD, \& Sugiarto, T. (2016). Malang Convention Center With Aesthetic Approach Space Frame Structure. Student Journal of Architecture, 4

[2] Fahrurrazi, D., 2011, the Geodetic Reference System, Gadjah Mada University Press, Yogyakarta.

[3] Gusty, FT (2014). Comparative Analysis of Structure Calculation shells of the Dome (Dome) Concrete Materials And Material Steel With Program. USU Civil Engineering Journal, 3 